\title{
CrystEngComm
}

Check for updates 7197

Received 11th July 2017,

Accepted 7th November 2017

DOI: $10.1039 / \mathrm{c} 7 \mathrm{ce} 01271 \mathrm{c}$

rsc.li/crystengcomm
Cite this: CrystEngComm, 2017, 19,

\section{Molecular dynamics simulations of aqueous glycine solutions $\uparrow$}

\author{
Yuriy G. Bushuev, (iD* Svetlana V. Davletbaeva (iD and Oscar I. Koifman (iD
}

\begin{abstract}
Simulations of glycine aqueous solutions have been carried out to study the effect of increasing solute concentration on the aggregation of glycine zwitterions. AMBER force field coupled with three atomic charge sets was employed for the simulations of the aqueous solutions. The number of glycine monomers in solutions rapidly decreases with increase in concentration. The properties of the glycine clusters in the solutions were studied. It was shown that the clusters are strongly hydrated entities with a liquid-like character. The residence lifetimes of water and glycine from the first solvation shell of glycine molecules were calculated. The lifetime values show that the clusters are highly dynamic solutes that change configuration within hundreds of picoseconds. We observed long-living pairs of glycine molecules, which move together in the solutions for ca. 1 ns. The effect of the electric-field-induced orientation of the highly polar glycine molecules in the clusters was studied. A preferential parallel arrangement of the molecules was observed as the distinctive feature of the $\gamma$-polymorph, which was crystallized from the solutions in a static electric field.
\end{abstract}

\section{Introduction}

Glycine is abundant in various proteins and enzymes. It is the smallest molecule among amino acids, which explains why glycine is an attractive object for investigation using experimental and computer simulation methods. In its crystalline states and in neutral aqueous solution, glycine exists in the zwitterionic form, $\mathrm{NH}_{3}{ }^{+}-\mathrm{CH}_{2}-\mathrm{COO}^{-}$. Three solid polymorphs, which are designated as $\alpha$-, $\beta$ - and $\gamma$-glycine, are detected under ambient conditions. A lot of factors affect glycine crystallization from a solution, such as $\mathrm{pH}$, additives, degree of supersaturation, solvent, impurities and seeding. These conditions determine the properties of the synthesized materials. Polymorphs have different physical and chemical properties including stability, solubility, melting point, compressibility and bioavailability. ${ }^{1,2}$

It has been established that $\gamma$-glycine is the most stable polymorph, whereas $\beta$-glycine has the highest free energy. ${ }^{3-5}$ Under ambient conditions and neutral $\mathrm{pH}$, an aqueous solution of glycine is predominantly crystalized as the $\alpha$-polymorph. The crystallization of a particular polymorph is poorly reproducible. Boldyreva et al. argued that the three

Institute of Chemistry of Macro Heterocyclic Compounds, Ivanovo State University of Chemistry and Technology, pr. Sheremetevskiy, 7, Ivanovo, 153000, Russia. E-mail: yuriyb2005@gmail.com

$\dagger$ Electronic supplementary information (ESI) available: Supporting figures, tables, snapshots of molecular configurations and DL_POLY files. See DOI: $10.1039 / \mathrm{c} 7 \mathrm{ce} 01271 \mathrm{c}$ polymorphs crystallize simultaneously. ${ }^{4}$ The nucleation and crystal growth of $\alpha$ - and $\gamma$-glycine from the same solution have been investigated experimentally. ${ }^{6}$ Little et al. ${ }^{6}$ concluded that the rate of nucleation is very sensitive to impurities, the conditions in which the samples are prepared and supersaturation. The least stable $\beta$-glycine polymorph crystalizes from aqueous solutions containing methanol or ethanol ${ }^{7}$ or from the gas phase via sublimation of the $\alpha$ or $\gamma$ forms in vacuum. ${ }^{8}$

It has been shown that intense nanosecond near-infrared laser pulses could induce supersaturated aqueous glycine solutions to crystallize into either the $\alpha$ - or $\gamma$-polymorph depending on the polarization state of the laser beam. ${ }^{9,10}$ This phenomenon is called non-photochemical laser-induced nucleation (NPLIN). Linearly polarized near-infrared laser pulses or a strong dc electric field applied to a supersaturated aqueous glycine solution induces the crystallization of $\gamma$-glycine. ${ }^{11}$ These methods could be used to control polymorph formation.

The difference in the size and shape of the glycine particles was observed when cooling and evaporative methods were used in their preparation with the addition of surfactants. ${ }^{12}$ Recent studies have indicated that under certain circumstances, in microemulsions and lamellar phases, the $\gamma$-glycine rather than the more common $\alpha$-glycine can be crystallized. ${ }^{13}$ It was proposed that the experimental results could be rationalized if it is postulated that molecular clusters binding to the interface were formed in the microemulsions. Glycine clusters can be captured by the charged surfactant 
monolayer. The structural and orientational specificity of the nucleation process must then be the result of the overall polarity of the clusters involved. However, the organization of the glycine clusters in solutions formed through the electricfield-induced alignment of molecules has not been studied using computer simulation methods, which could provide information regarding the structure of the solutions.

Computer simulations of glycine polymorphs and their aqueous solutions have been performed. ${ }^{14-21}$ Classical molecular dynamics (MD) simulations are based on using of force fields, which usually include intra- and inter-molecular terms. The results of the computer simulations depend on the employed force field. To minimize this influence, the calculations should be repeated with different force fields. Various sets of potentials have been proposed for the calculation of glycine interactions. AMBER force field was particularly designed for the simulation of amino acids, proteins and large organic molecules. ${ }^{22}$ It has been widely used for simulations over the last several decades. To calculate the energy of the Coulombic interactions, numerous atomic charge sets are employed. For a specific compound, the atomic charges are chosen according to results of quantum mechanics calculations or they are fitted to reproduce the experimental properties of solids, liquids or solutions. Systematic investigations of the applicability of different force fields for the simulation of glycine aqueous solutions have been published. Cheong and Boon ${ }^{21}$ used Charmm27, AMBER, OPLS and GROMOS force fields with six charge sets adopted for the calculation of the electrostatic interactions. The authors concluded that the AMBER force field coupled with CNDO charges is the optimal force field to be used for simulation studies of the $\alpha$-glycine crystal growth.

Understanding the crystal growth mechanism is valuable for pharmaceutical industry and drug design. Glycine is a major precursor in the biosynthesis of porphyrins. New functional materials based on macro-heterocyclic compounds and their complexes have been widely used in various fields of science, technology and medicine. Of particular interest is the study of porphyrin complexes with various amino acids and biomolecules. ${ }^{23,24}$ For this purpose, the properties of glycine solutions should be investigated. It has been proposed ${ }^{25}$ that among a set of compounds, such as calcium carbonate, calcium phosphate and silica, amino acids form prenucleation clusters (PNCs) in aqueous solutions, which have specific properties. The PNCs are solute precursors to the phases formed from a homogeneous solution, but, to date, the actual scenario of nucleation and crystallization from glycine aqueous solutions is unknown.

The goal of this article is the study of the glycine clusters formed in aqueous solutions at different concentrations. The size distribution and radii of gyration are the basic properties of clusters. To characterize the stability of the clusters, the residence lifetimes were calculated: (a) for water from the first hydration shells of hydrophilic moieties of a glycine and (b) for glycine molecules forming clusters. To shed light on the molecular mechanism of the NPLIN phenomenon, the structure of a saturated solution of glycine under an applied dc electric field was investigated.

\section{Methods}

Classical molecular dynamics (MD) simulations were employed to study the aqueous solutions of glycine. All calculations were performed using the DL_POLY Classic 1.9 pro$\operatorname{gram}^{26,27}$ in the isotropic $N p T$ ensemble at $T=300 \mathrm{~K}, 350 \mathrm{~K}$ and $p=0.1 \mathrm{MPa}$. The Nosé-Hoover thermostat and barostat, designed by Melchionna et al., ${ }^{28}$ was employed with relaxation times of 0.1 ps $(T)$ and 1 ps $(p)$ as previously recommended. ${ }^{29}$ An example of the typical time-dependence of volume and energy is presented in Fig. S1. $\dagger$ The integration time-step was $1 \mathrm{fs}$. The cubic boxes, taken with the periodic boundary conditions, contained 1200 water and 12, 36 and 72 glycine molecules. The last composition corresponds to the concentration of a saturated solution of glycine $(m=$ $\left.3.33 \mathrm{~mol} \mathrm{~kg}{ }^{-1}\right) .{ }^{30} \mathrm{~A}$ cut-off radius of $12 \AA$ was used for both the non-bonded and electrostatic interactions. Long-range electrostatic interactions were treated using the Ewald method. The relaxation period was $c a$. $1 \mathrm{~ns}$. For trajectory analysis, the structures were saved every 10000 time-steps $(\Delta t$ $=10 \mathrm{ps}$ ), however, the interval was of $1 \mathrm{ps}$ when the diffusion coefficients were calculated. The simulation time in each productive run exceeded $3 \mathrm{~ns}$ (Table $\mathrm{S} 1 \dagger$ ). A cluster analysis was performed on the simulation snapshots of the liquids to determine how the glycine molecules tend to associate with each other in the aqueous solutions. The Edvinsson algorithm ${ }^{31}$ was used to calculate cluster statistics.

AMBER force field, which is well-proven in the simulation of large organic molecules, was applied to calculate the intraand inter-molecular interactions of glycine. ${ }^{22}$ The chargecharge interactions between atoms in a molecule separated by 3 bonds (1-4 interactions) were scaled by a factor of $1 / 1.2$ and the Lennard-Jones interactions by a factor of 0.5 . To calculate the interactions of the water molecules, the SPC/Fw potential $^{32}$ was selected, which takes into account the flexibility of the water molecules. We employed the DL_FIELD version 3.2 program package, which simplifies the procedure of creating the FIELD file required for the DL_POLY program.

Based on the results of the quantum-chemical calculations, various charge models were proposed for glycine. ${ }^{14,23,24,29,39,40}$ In the present study, the three charge sets,

Table 1 Atomic charges for the atoms in zwitterionic glycine $\left(\mathrm{H}_{3} \mathrm{~N}-\mathrm{CH}_{2}-\right.$ $\left.\mathrm{CO}_{2}\right)$

\begin{tabular}{lccc}
\hline Atom & ${\mathrm{B} 3 \mathrm{LYP}^{a}}^{a}$ & $\mathrm{CNDO}^{b}$ & $\mathrm{mB3LYP}$ \\
\hline $\mathrm{N}$ & -0.386 & 0.021 & -0.436 \\
$\mathrm{H}_{\mathrm{N}}$ & 0.34 & 0.19 & 0.34 \\
$\mathrm{C}_{\mathrm{H}}$ & 0.022 & -0.021 & 0.072 \\
$\mathrm{H}_{\mathrm{C}}$ & 0.064 & 0.0315 & 0.064 \\
$\mathrm{C}_{\mathrm{O}}$ & 0.9 & 0.375 & 0.9 \\
$\mathrm{O}$ & -0.842 & -0.504 & -0.842
\end{tabular}

${ }^{a}$ Ref. $16 .{ }^{b}$ Ref. $33 .^{c}$ This work. 
summarised in Table 1, were used for the MD simulations. The ESP (the Merz-Kollman scheme) electrostatic partial charge method was used to derive the B3LYP partial charges. ${ }^{16}$ The CNDO charge set was calculated from gross Mulliken populations. ${ }^{33}$ Despite the arbitrary nature of the method, these atomic charge sets were used in the calculations and a good reproduction of experimental properties of the crystals and aqueous solutions of glycine was demonstrated. $^{21,34}$ We slightly corrected the B3LYP charge set. According to our calculations, the modified B3LYP (mB3LYP) charge set predicts $\alpha$ - and $\gamma$-glycine structures better than the other sets. Any force field used for flexible glycine molecules contains terms that take into account the rotations of the $-\mathrm{COO}$ and $-\mathrm{NH}_{3}$ groups. Thus, the original atomic charges proposed for a rigid molecule were slightly corrected to eliminate the difference in the charges of the $\mathrm{O}$ and $\mathrm{H}$ atoms when the molecule adopts various conformations. The dipole moment of a glycine molecule was calculated to be 10.3, 13.8 and 13.5 D for the CNDO, B3LYP and MB3LYP sets, respectively. The results of the $a b$ initio MD simulation of an aqueous glycine solution demonstrate that the dipole moment of a glycine molecule increases by about $40 \%$ due to polarization effects and it was around $16.5 \mathrm{D}^{23}$ To investigate a possible dependence of the results on the employed force field, independent simulations of the systems were carried out with three charge sets. The parameters of the force field are presented in ESI. $\dagger$

\section{Results and discussion}

\section{Density and self-diffusion coefficients}

To test the reliability of the models, the densities and diffusion coefficients were calculated for the aqueous solutions of glycine. In the equilibrium MD simulation, the self-diffusion coefficient $(D)$ was computed by taking the slope of the mean-squared displacement (MSD) at long time

$$
D=\frac{1}{6 N} \lim _{t \rightarrow \infty} \frac{d}{d t}\left\langle\sum_{i=1}^{N}\left(r_{i}(t)-r_{i}(0)\right)^{2}\right\rangle,
$$

where $N$ is the number of molecules, $r_{i}(t)$ is the position of the molecule $i$ at time $t$. The values of $D$ were obtained from the slope of a linear fit of the respective MSD data, which were calculated from the stored trajectories. Examples of the MSD plots are shown in Fig. S2 and S3. $\dagger$

The experimental ${ }^{35}$ and calculated densities are plotted in Fig. 1a. The density of SPC/Fw water was about $1 \%$ higher than the experimental value, therefore the difference between the calculated and experimental density of pure water could be taken into consideration. The presented results demonstrate an almost linear increase in the density of the solution with increase in concentration of glycine. The slopes of the fitting lines, corresponding to the B3LYP and MB3LYP models, slightly differ from that obtained experimentally. This is a typical situation observed for several force fields. The force fields GAFF, OPLS and Charmm with default atomic charge sets predict higher density of solutions with respect to the experimental values. ${ }^{21}$ By choosing the appropriate charge set, it is possible to reach a better agreement with the experimental data, but it does not mean that other properties of the aqueous solutions would be effectively reproduced.

Fig. 1b shows the variation of the calculated self-diffusion coefficients of glycine and water with the solute concentration. The diffusion coefficient of water molecules is more than two times larger than the diffusion coefficient of glycine in solution. The diffusivity of water and glycine decreases with increase in concentration. The CNDO model demonstrates a weaker concentration dependence of the water diffusivity. The experimental ${ }^{36}$ self-diffusion coefficients of glycine in the solutions were taken for comparison with the results of our calculations. The calculated values are slightly higher (CNDO) or lower (B3YLP and MB3LYP) than the experimental values.

For pure water the calculated $D=(2.42 \pm 0.08) \times 10^{-9} \mathrm{~m}^{2}$ $\mathrm{s}^{-1}$ (SPC/Fw), which is larger than the experimental $D=2.3 \times$ $10^{-9} \mathrm{~m}^{2} \mathrm{~s}^{-1} .{ }^{37}$ The weak and approximately linear dependence of water diffusivity on the concentration has been reported for the AMBER force field with other charge sets. ${ }^{38}$ Thus, the
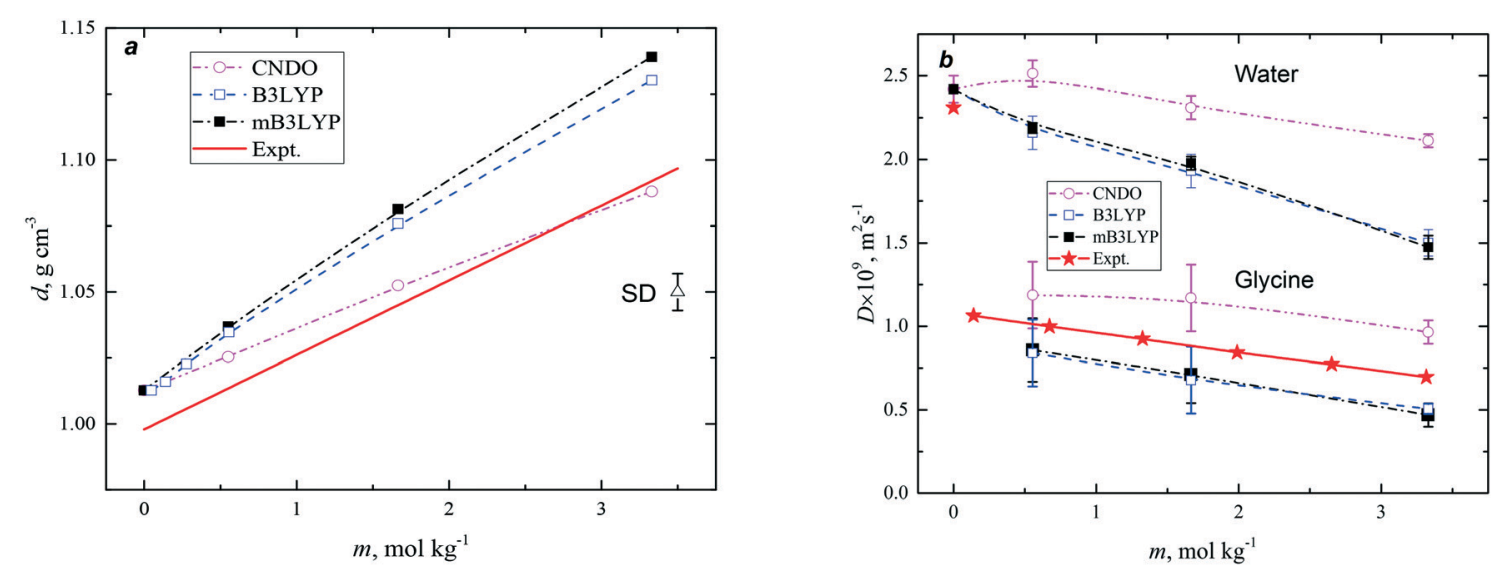

Fig. 1 (a) Density of the aqueous solutions and (b) diffusion coefficients of water and glycine calculated using the three charge sets. Error bar represents one standard deviation (SD). The lines connecting the data points are drawn to guide the eye. 
presented models of the solutions are in agreement with the experimental data. The variation observed for the calculated properties are in accordance with the results of previous simulations, where other force fields were tested. ${ }^{25,29,38}$ The experimental diffusion coefficients obtained for the glycine in solutions are slightly over- (CNDO) or under-estimated (B3LYP and mB3LYP) in the computer models and these effects correlate with the dipole moment of the glycine molecules. In solution, the diffusivity of glycine and water decreases with an increase in the dipole moment. We could expect that for better correspondence with the experimental data, the model of the glycine molecule should have a dipole moment of about $12 \mathrm{D}$.

\section{Correlation functions and running coordination numbers}

Radial distribution functions (RDFs) are usually used for the description of disordered systems. The structure of the hydration shells of glycine molecules has been discussed previously. ${ }^{14,15,39-41}$ The present investigation is primarily focussed on the study of the aggregation of glycine in the solutions. Therefore, to avoid a repetition of the results, only two functions, which characterize the distribution of nitrogen atoms, $g_{\mathrm{NN}}$, and oxygen atoms with respect to the nitrogen atoms, $g_{\mathrm{NO}}$ as well as the corresponding running coordination numbers, were calculated to analyse the structures formed by glycine molecules in the aqueous solutions.

These functions are presented in Fig. 2 and S4. $\dagger$ They depend on the model and concentration of the solutions; however, these functions demonstrate similar behaviour. Two overlapping peaks for $g_{\mathrm{NN}}$ were observed at $r<6.5 \AA$ and correspond to the minimum two types of atomic coordination in the first solvation shell of each nitrogen atom. The $N_{\mathrm{NN}}$ functions show that the aggregation of glycine molecules increases with increase in concentration and was larger for the CNDO model at any concentration despite having the smallest dipole moment of the molecule. The $g_{\text {NO }}$ functions (Fig. S4†) exhibit the first peak at ca. $3 \AA$, corresponding to the formation of intermolecular hydrogen bonds between hy- drogens of the amino group and oxygens of nearest glycine molecules. The running coordination numbers, $N_{\text {NO }}$, show larger molecular aggregation when the CNDO charge set was used for the simulations.

The molecular configurations, presented in Fig. S5 and $\mathrm{S} 6, \dagger$ show that various types of glycine aggregation are possible at $m=3.33 \mathrm{~mol} \mathrm{~kg}^{-1}$. The two small glycine clusters are presented in Fig. S7. $\uparrow$ The first cluster is a compact threemember ring, while the larger cluster has a complex structure, where a compact 'head' is connected to a long 'tail'.

It was shown ${ }^{11}$ that a strong static electric field $(E=6 \times$ $10^{5} \mathrm{~V} \mathrm{~m}^{-1}$ ) applied to supersaturated aqueous glycine solutions (SS is ca. 2.0) results in the nucleation of the $\gamma$-polymorph. We performed simulations of the concentrated aqueous solutions under a dc electric field at $E=10^{3}$ and $10^{4}$ $\mathrm{V} \mathrm{m}^{-1}$. A preferable orientation of the glycine molecules along the electric field was observed. The average cosine of the angle between the vectors connecting the carbon atoms $\left(\mathrm{H}_{2} \mathrm{C} \rightarrow\right.$ $\mathrm{CO}_{2}$ ) in the glycine molecules was calculated. The results are presented in Fig. 3 and $\mathrm{S} 8 \uparrow$ for the CNDO and MB3LYP force fields. The cosine value increases with the increase in electric field strength and becomes close to 1 . The parallel arrangement of the $\mathrm{C}-\mathrm{C}$ vectors is a distinctive feature of the $\gamma$-polymorph (Fig. S9†). The temperature has little effect on the orientation of the glycine molecules in these electric fields. The crystallization of glycine is a long process and cannot be reproduced using computer simulations; however, the results obtained in our study show the orientational ordering of the glycine molecules in the solutions when a strong electric field was applied. This corresponds to experimental observations.

\section{Finite cluster size distributions and radius of gyration}

RDFs provide general information on the correlations between the atomic positions in liquids. The types of molecular association can be studied using cluster analysis, which is widely used in percolation theory. ${ }^{42}$ Phase transitions and percolation have many similarities. The percolation
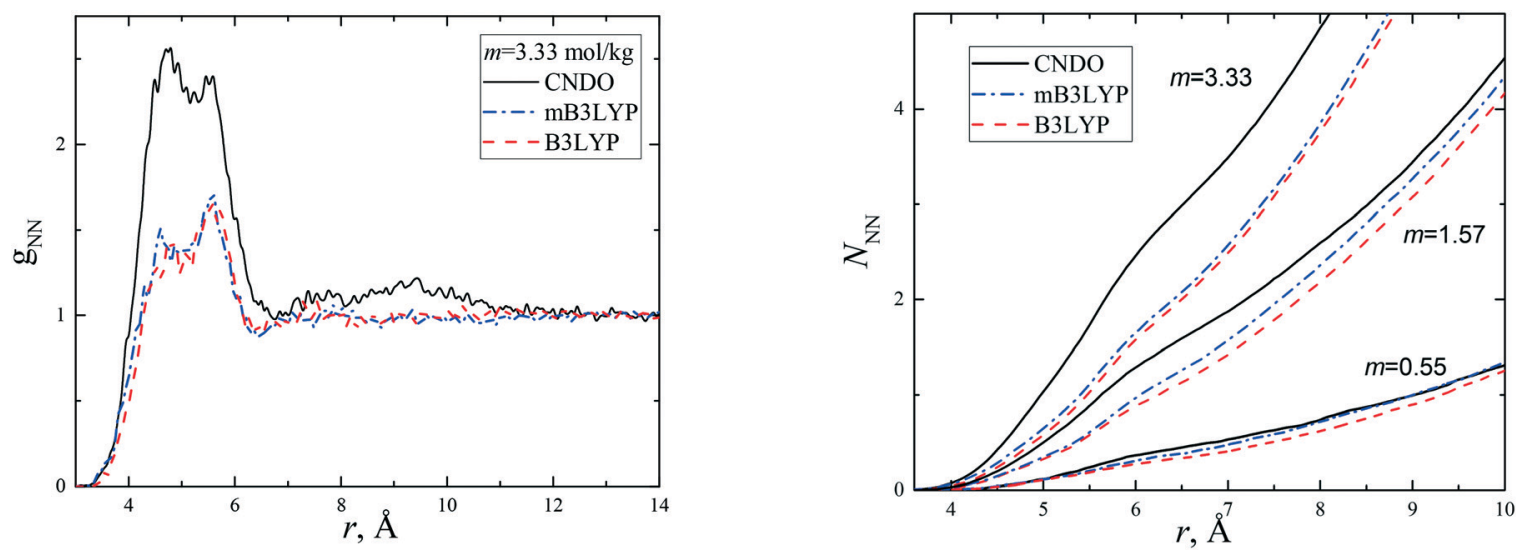

Fig. 2 Radial distribution functions, $g_{\mathrm{NN}}(r)$, and running coordination numbers, $N_{\mathrm{NN}}(r)$, obtained for the nitrogen atoms of glycine in aqueous solutions with various solute concentrations $(m)$. 


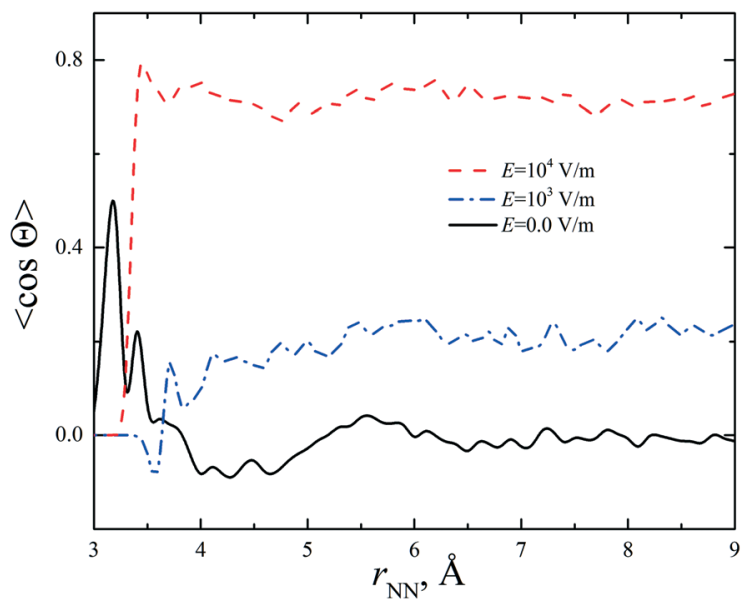

Fig. 3 Average cosine of the angle between vectors connecting the carbon atoms $\left(\mathrm{H}_{2} \mathrm{C} \rightarrow \mathrm{CO}_{2}\right)$ in two glycine molecules calculated without and with a dc electric field $\left(E=10^{3}\right.$ and $\left.10^{4} \mathrm{~V} \mathrm{~m}^{-1}\right)$ applied to the solution at $m=3.33 \mathrm{~mol} \mathrm{~kg}^{-1}$.

threshold, $p_{\mathrm{c}}$, is a point of singularity for many properties and these properties show a universal scaling behavior around percolation. In particular, the number of finite clusters decreases with the increase in cluster size, $s$, according to $n_{s} \sim s^{-\tau}$, where $\tau=2.189$ is a universal constant and $s$ is the number of molecules in the cluster. ${ }^{43}$ The radius of gyration, $R_{\mathrm{g}}$, is a fundamental characteristic of the finite clusters calculated according to the formula:

$$
R_{\mathrm{g}}^{2}(s)=\frac{\left[\sum_{i=1}^{s}\left(r_{i}-\langle r\rangle\right)^{2}\right]}{s},\langle r\rangle=\sum_{i=1}^{s} r_{i} / s
$$

where the vectors $r_{i}$ define the positions of the monomers in the cluster. Near the percolation threshold, $R_{\mathrm{g}}$ increases with increase in size $(s)$ as a power function, $R_{g}(s) \sim s^{1 / d_{\mathrm{f}}}$, where $d_{\mathrm{f}}$ $=2.523$ is the fractal dimension of the clusters near the percolation threshold.

The clustering of glycine molecules in an aqueous solution was studied previously. ${ }^{20,39}$ Two specific hydrogen bond criteria, a $\mathrm{H} \cdots \mathrm{O}$ distance of $2.2 \AA$ and a minimum $\mathrm{N}-\mathrm{H} \cdots \mathrm{O}$ angle of $140^{\circ}$ as well as $2.2 \AA$ per $160^{\circ}$ were used in analysis of the clustering of glycine molecules from the MD simulations. In the aqueous solutions the hydrogen bonds are constantly forming and breaking only to form once again. It was shown $^{20,39}$ that the lifetime of the hydrogen bonds was about of 1-4 ps. Due to the coordinated collective movement of the molecular aggregates, the glycine molecules stay close to each other for a longer time. This type of movement has not been studied to date.

To avoid a repetition of the same calculations and to obtain new information, we did not analyse the hydrogen bonding; thus, in the present study, a criterion of mutual proximity of glycine molecules was applied. The position of the nitrogen atom was taken as a center of a monomer $(s=1)$ and the glycine molecule was considered belonging to a clus- ter if the distance between the nitrogen atom and any other nitrogen in the cluster was less than $6 \AA$. This distance approximately corresponds to the first coordination shell of glycine molecule (Fig. 2). Clearly, the cluster properties depend on the value of the threshold distance; however, this is not very important for a general characterization of the aggregation of glycine molecules in the solutions.

In all the investigated cases, spanning glycine clusters were not formed in the simulation boxes at $E=0 \mathrm{~V} \mathrm{~m}^{-1}$. All the observed clusters were finite. This means that even at $m$ $=3.33 \mathrm{~mol} \mathrm{~kg}^{-1}$ the systems are located below the percolation threshold and the indexes could deviate from the universal critical values. A large cluster comprised of 40 molecules is presented in Fig. 4. Bulky fragments containing 4-6 molecules connected by chains are observed.

The cluster size distributions, $n_{\mathrm{s}}(s)$, were calculated from our simulations for $m=3.33 \mathrm{~mol} \mathrm{~kg}^{-1}$ (72 glycine molecules in the simulation box) and they are depicted in Fig. 5 and S10. A broad spectrum of the cluster sizes was observed in the saturated solution. The presented distributions depend on the charge set used. All the systems are far from the percolation threshold; however, when the CNDO charge set was used, the slope of the fitting line was closer to the value of universal exponent, $\tau=2.19$. The results of the small-angle neutron scattering (SANS) experiments show that the glycine aggregates in the solutions can be described by relatively broad distributions of both size and shape. ${ }^{41}$ The set of clusters presented in Fig. S5-S7† show that the clusters have various shapes. Thus, the results of our computer simulations are in agreement with the results of the SANS experiments.

Temperature has little effect on the distribution as shown in Fig. S11. However, a strong electric field supports cluster formation. The number of small glycine clusters $(s<7)$ decreases slightly at $350 \mathrm{~K}$ and $E=10^{4} \mathrm{~V} \mathrm{~m}^{-1}$, but the number of large clusters increases significantly. Percolated chain- and strip-like glycine clusters are observed in the simulation box. Some of these are shown in Fig. S12.†

The probabilities of finding a glycine molecule in a monomeric state $(s=1)$ and small clusters $(s<13)$ are presented
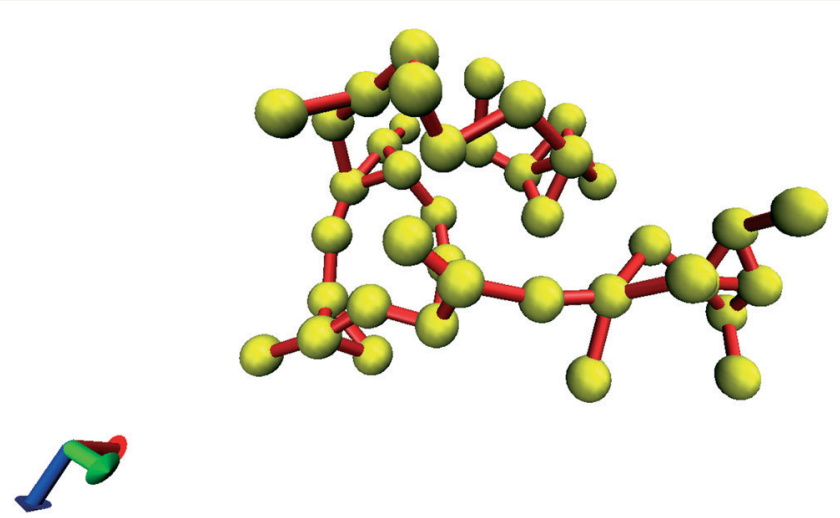

Fig. 4 The finite cluster consisting of 40 glycine molecules, $\left(R_{\mathrm{g}}=13\right.$ Å). Nitrogens are shown as yellow balls. Red sticks connect the nearest nitrogen atoms. 


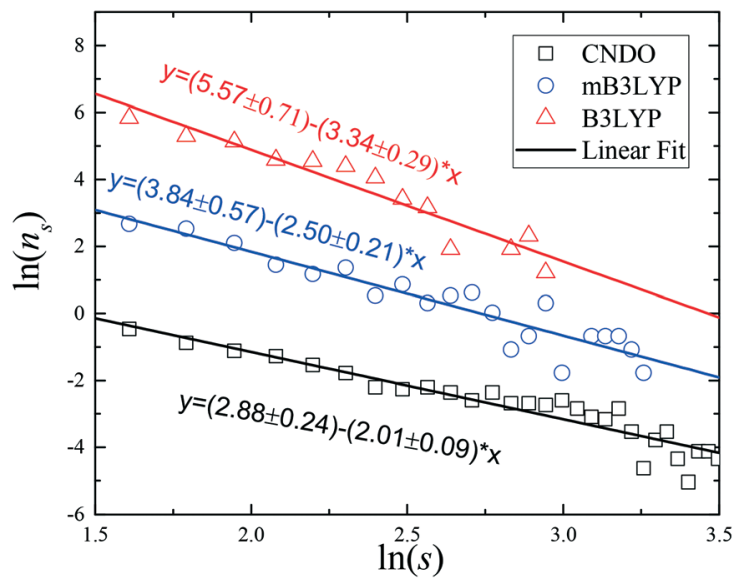

Fig. 5 Number of glycine clusters, $n_{s}$, in the saturated aqueous solution ( $m=3.33 \mathrm{~mol} \mathrm{~kg}^{-1}$ ) as a function of cluster size, $s$. For clarity the plots for the $\mathrm{mB} 4 \mathrm{LYP}$ and B3LYP charge sets are shifted vertically to $\ln \left(n_{s}\right)+3$ and $\ln \left(n_{s}\right)+6$, respectively.

in Fig. 6. The fraction of the monomers drops from ca. $80 \%$ at $m=0.55 \mathrm{~mol} \mathrm{~kg}^{-1}$ to $25-35 \%$ in the saturated solution, whereas the fractions of glycine molecules belonging to dimers and trimers weakly depends on the solute concentration. Small clusters prevail in all the models, but the system computed with the B3LYP charge set has a larger amount of small clusters $(s<6)$. The calculated fractions are consistent with the previously reported results obtained using a different force field and a criterion of a molecular aggregation. ${ }^{41}$ It is noteworthy that a significant variation in the fraction of glycine molecules in the monomers, dimers and trimers was not observed in a temperature range from 20 to $50{ }^{\circ} \mathrm{C} .{ }^{41}$

An average cluster size and an average radius of gyration are the first moments of the distribution, $n_{s}$ :

$$
\langle S\rangle=\sum_{s} s n_{s} / \sum_{s} n_{s} ;\left\langle R_{\mathrm{g}}\right\rangle=\sum_{s} R_{\mathrm{g}}(s) n_{s} / \sum_{s} n_{s},
$$

where $n_{s}$ is the number of finite clusters, $R_{\mathrm{g}}(s)$ is the average radius of gyration of clusters containing $s$ glycine molecules. In the presented range, the average size of clusters increases with concentration as it is shown in Fig. 7. At high concentrations, the glycine molecules are more self-associated in the CNDO model. This conclusion is in accordance with the results of the analysis of the RDFs presented in Fig. 2. The minimal average cluster sizes were observed for the B3LYP set. Depending on the model, in a saturated solution an average cluster is composed from 5 (B3LYP) to 12 (CNDO) molecules. At low concentration, small clusters are predominantly formed and the average size corresponds to the dimer.

The plots of $R_{\mathrm{g}}(s)$ calculated from our simulations are depicted in Fig. 8 and S13.† The slopes of the fitting lines deviate from the universal scaling law $\left(1 / d_{\mathrm{f}}=0.396\right)$ because the systems are far from the percolation threshold. The larger values of the slopes indicate that the clusters grow in 3-D space faster than the clusters near the percolation threshold. The fractal dimension of the clusters was $c a$. 2.0-2.1. The radii of gyration calculated at $350 \mathrm{~K}$ with and without an external electric field are presented in Fig. S14. $\dagger$ The cluster size distribution and radii of gyration are the basic properties of the system. They depend on the model used; however, the temperature and strong electric field do not affect these distributions. The number of large clusters increases with increase in the electric field and they have large radii.

The nucleation and crystallization of glycine from a supersaturated solution were studied using a small angle X-ray scattering (SAXS) method. ${ }^{44,45}$ In order to achieve supersaturation, two procedures of cooling were used. The measured average radius of gyration monotonically increased upon cooling from 3.38 to $4.0 \AA^{44}$ In the later experiment, ${ }^{45}\left\langle R_{\mathrm{g}}\right\rangle$ increased from 2.72 to $3.2 \AA$ during the initial $15 \mathrm{~min}$ of cooling and then stayed near $3.2 \AA$ for the next $40 \mathrm{~min}$. In both experiments, the average radius of gyration reached a maximum value of $4.24 \AA$, followed by a decrease to $4.02 \AA$.
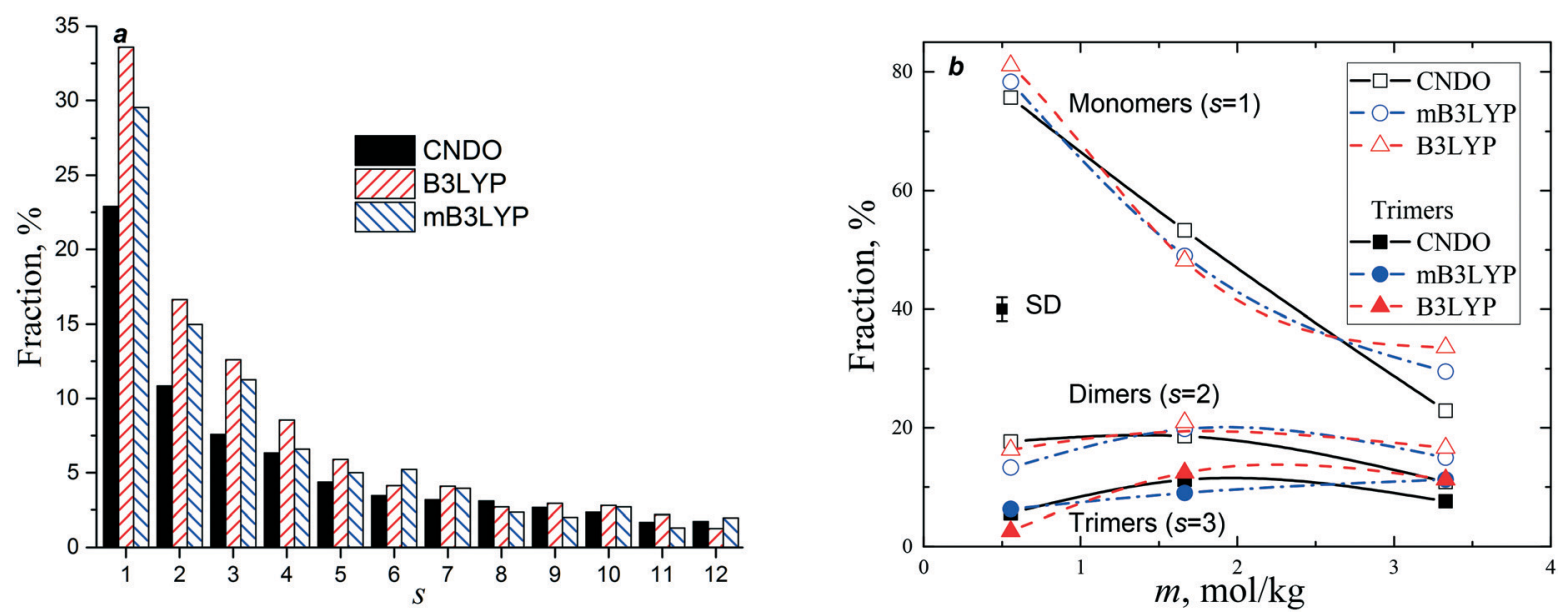

Fig. 6 Fraction of glycine molecules belonging to clusters: (a) vs. cluster size at $m=3.33 \mathrm{~mol} \mathrm{~kg}^{-1}$. Data for $s>12$ are not shown for clarity; (b) vs. concentration of the solution for $s<4$. Error bar is one standard deviation (SD). The lines connecting the data points are drawn to guide the eye. 


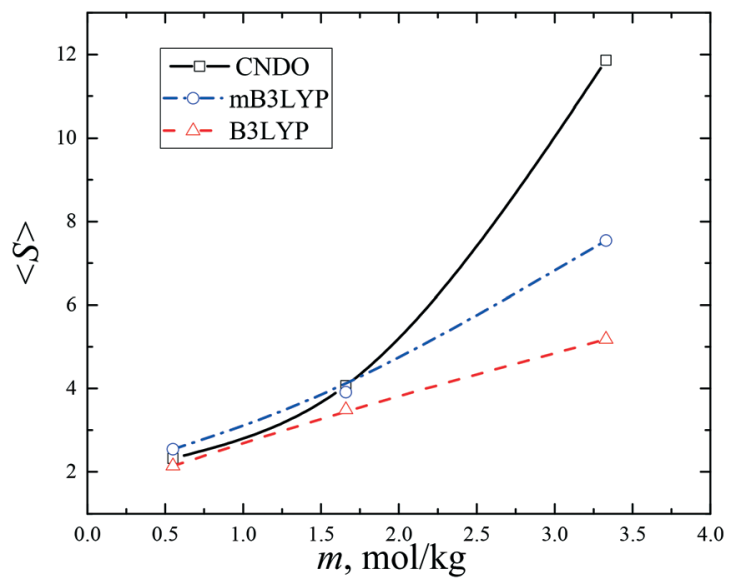

Fig. 7 Average sizes $\langle S\rangle$ of the glycine clusters in the aqueous solutions. The lines connecting the data points are drawn to guide the eye.

These variations in $\left\langle R_{\mathrm{g}}\right\rangle$ are in good agreement with data presented in Fig. 8b. Our calculation predicts that, depending on the model used, in a saturated solution, the radius varies from 3.7 (B3LYP) to $4.8 \AA$ (CNDO). The average radius of gyration increases with the increase in concentration of glycine at a fixed temperature or with increase in the supersaturation in the cooling processes. Simulations using the mB3LYP force field demonstrate that $\left\langle R_{g}\right\rangle$ increases from $3.8 \AA$ to $4.5 \AA$ $(4.3 \AA$ at $T=350 \mathrm{~K})$ in the strong electric field $\left(E=10^{4} \mathrm{~V}\right.$ $\left.\mathrm{m}^{-1}\right)$.

It has been proposed, ${ }^{44,45}$ that the crystallization initiates at $\left\langle R_{\mathrm{g}}\right\rangle=4.24 \AA$. According to our calculations, this value corresponds to $s=4$, but this does not indicate that the critical nucleus is very small or tetramers predominate in the solution. The distribution of $R_{\mathrm{g}}(s)$ is broad and asymmetric (Fig. S13 and S14 $\dagger$ ). At the percolation threshold, clusters of all sizes, including the infinite cluster, are simultaneously present in the system. At $E=0 \mathrm{~V} \mathrm{~m}^{-1}$ the largest clusters observed in our simulations contain 44 glycine molecules. According to a probabilistic approach in the frame of classical nucleation theory, nucleation is a stochastic process. ${ }^{46,47}$ The probability of the formation of large clusters decreases with increase in size as $s^{-\tau}$ (Fig. 5). In the laboratory, crystals usually start to grow with a time lag of several hours after the supersaturated solution is prepared. If classical nucleation theory is applicable to the description of crystallization from glycine aqueous solutions, we could expect that a critical nucleus would contain hundreds of glycine molecules.

\section{Residence lifetimes}

To describe the collective molecular movement in the aqueous solutions, the distribution of the residence lifetimes of water molecules from the first hydration shells of the hydrophilic $-\mathrm{NH}_{3}$ and $-\mathrm{COO}$ groups was calculated. The boundaries of the shells were selected according to positions of the first minima of the RDFs as shown in Fig. S15. $\dagger$ The hydration numbers strongly depend on the threshold interatomic distances. At $m=3.33 \mathrm{~mol} \mathrm{~kg}^{-1}$, the first hydration shell of the $\mathrm{NH}_{3}$ group $\left(r_{\mathrm{NOw}}<3.4 \AA\right)$ contains an average 4.0 (mB3LYP, B3LYP) or 3.1 (CNDO) water molecules, which are close to the experimental value of $3.0 \pm 0.6 .{ }^{39}$ The hydration shell of the -COO group $\left(r_{\mathrm{COw}}<4.0 \AA\right)$ contains 7.2 (mB3LYP, B3LYP) and 5.2 (CNDO) molecules. Some molecules, belonging to the hydration shell of the hydrophobic $-\mathrm{CH}_{2}$ group, occur in the hydration shell of the - $\mathrm{COO}$ group. During the residence lifetime, the glycine and water molecules are moving together and the water molecules belong to the first hydration shell of the glycine molecule. Due to diffusion and a molecular exchange between hydration shells, the water molecules leave early or late from the first shell.

A hydrogen bond criterion typically has two adjustable parameters, namely, the interatomic distance and the hydrogen bond angle. The residence lifetimes of the water-glycine aggregates differ from the lifetimes of hydrogen bonds, which
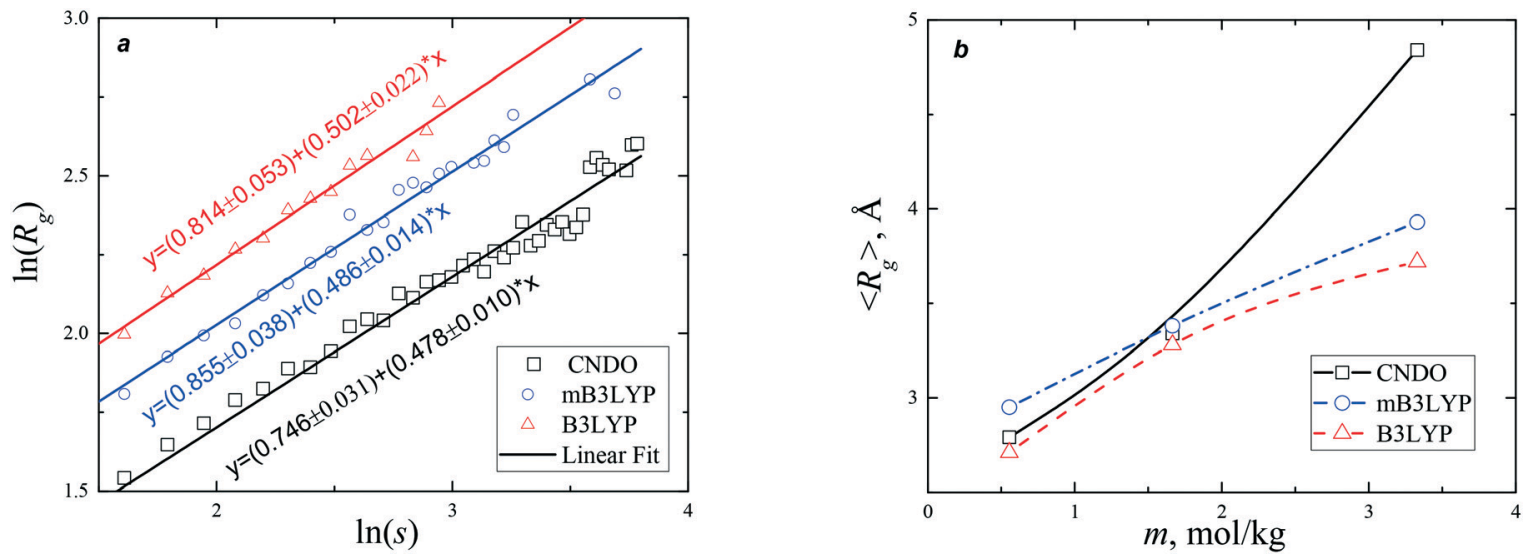

Fig. 8 (a) Radius of gyration, $R_{\mathrm{g}}$, obtained for the glycine clusters in a saturated aqueous solution ( $m=3.33 \mathrm{~mol} \mathrm{~kg}^{-1}$ ), as a function of the cluster size, s. For clarity the plots for $\mathrm{mB} 3 \mathrm{LYP}$ and B3LYP are shifted vertically to $\ln \left(R_{\mathrm{g}}\right)+0.2$ and $\ln \left(R_{\mathrm{g}}\right)+0.4$, respectively. (b) Average radius of gyration, $\left\langle R_{\mathrm{g}}\right\rangle$, as a function of the glycine concentration in an aqueous solution. The lines connecting the data points are drawn to guide the eye. 
take place on a timescale of several picoseconds ${ }^{20,38}$ because the threshold distances are larger than values usually used as hydrogen bond criteria and the mutual orientation of the molecules are not taken into consideration.

To calculate the residence lifetimes, we analysed snapshots of the systems stored with a step of $10 \mathrm{ps}$. A list of water molecules, within the cut-off distance $\left(r_{\text {Now }}<3.4 \AA\right.$ or $r_{\mathrm{COw}}<4.0 \AA$ ) from each $-\mathrm{NH}_{3}$ or -COO group of any glycine molecule, was composed at each time step $t_{1}$. Then, the lifetime was calculated according to the equation: $t=t_{\mathrm{e}}-t_{\mathrm{s}}$, where $t_{\mathrm{e}}$ is the time when the water molecule leaves the first hydration shell and $t_{\mathrm{s}}$ is the time of appearance of the molecule in the shell.

The distributions of the fractions $F$ of water molecules versus the lifetime are shown in Fig. S16. $\uparrow$ To fit the calculated data, the model of exponential decay was applied according to the equation:

$$
F(t)=\sum_{i=1}^{n} A_{i} \exp \left(-\frac{t}{\tau_{1}}\right),
$$

where $\tau_{i}$ is the mean lifetime. For the water-glycine associates, statistical analysis showed that in most cases, only one term was enough $(n=1)$ to approximate the calculated data. The mean lifetime depends on the glycine concentration and the charge set. These dependences are shown in Fig. 9. The mean residence lifetimes vary from 5 to 18 ps. They are longer for water molecules from the first hydration shell of carbons than of nitrogen atoms. At each concentration, the shortest mean lifetime was observed for the CNDO charge set. In all cases the values of the lifetimes show an approximately linear increase with increase in the concentration of glycine in the aqueous solutions. This correlates with the decreasing water diffusion coefficient with increase in concentration (Fig. 1b). The weaker hydration of glycine molecules promotes their aggregation. To approximate the distribution (eqn (4)) at

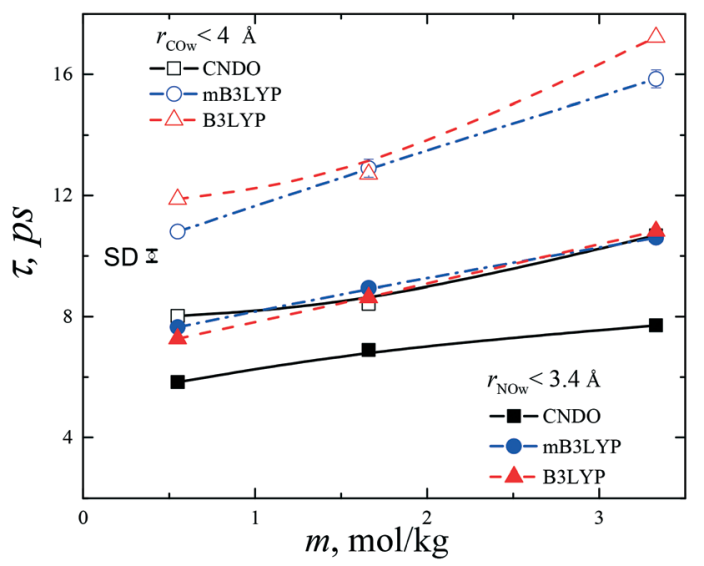

Fig. 9 Mean residence lifetimes for water molecules from the first hydration shells of $-\mathrm{COO}$ and $-\mathrm{NH}_{3}$ groups in the glycine molecules. Error bar is one standard deviation (SD). The lines connecting the data points are drawn to guide the eye. high temperature and in the presence of a strong electric field, two pairs of parameters were needed $(n=2)$. Shortand long-living glycine-water pairs were observed. The residence lifetime for the long-living water near the -COO groups increases with the electric field from 29 ps to 34 ps at $T=300 \mathrm{~K}$ and decreases with temperature to $19 \mathrm{ps}$ and $23 \mathrm{ps}$ at $T=350 \mathrm{~K}, E=0$ and $10^{4} \mathrm{~V} \mathrm{~m} \mathrm{~m}^{-1}$, respectively. The same tendency was observed for water molecules near the $-\mathrm{NH}_{3}$ groups whose residence lifetimes decrease with increase in temperature by $c a .5 \mathrm{ps}$ and increase with an increase in the electric field strength by ca. 4 ps.

Other behaviours of residence lifetimes were observed for glycine molecules in clusters formed at high concentrations. These lifetimes characterize the stability of the clusters and they differ from the glycine-glycine hydrogen bond lifetime. The hydrogen bond formation and breaking processes have been studied recently. ${ }^{38}$ In the cited study, the exponential decay model was not applied to fit the hydrogen bond correlation function.

To calculate the glycine-glycine residence lifetimes, the cut-off distance between the nitrogen atoms was selected to be $6 \AA$. Various structures of the glycine dimers were found in the aqueous solutions and crystalline polymorphs. A statistical analysis of the distributions, $F(t)$, (Fig. S17 $\dagger$ ) show that two terms $(n=2)$ in eqn (4) should be used to approximate the calculated data. This indicates that the short- and long-living pairs of the glycine molecules were formed in the solutions. At the highest concentration, the systems contain 72 glycine molecules in the simulation boxes and the monomers were not taken into consideration when residence lifetimes were calculated. It is difficult to estimate the number of long-living pairs due to poor statistics of events (Fig. S17c $\dagger$ ). Nevertheless, the mean lifetimes were calculated as 14.3 and 112.4 ps; 12.3 and $96.8 \mathrm{ps} ; 13.1$ and $108.3 \mathrm{ps}$ for the B3LYP, MB3LYP and CNDO models, respectively. For the mB3LYP model at $350 \mathrm{~K}$ and $E=10^{4} \mathrm{~V} \mathrm{~m}^{-1}$ the residence times increase to 13.2 and 109.1 ps. The short-living glycine pairs of the molecules have mean lifetimes close to the lifetimes of water molecules near the hydrophilic fragments of the glycine molecules. However, we observed pairs of glycine molecules, which moved together in the solution for longer than $1 \mathrm{~ns}$. Restructuring of the glycine clusters was observed during visualization of the molecular configurations, but some 'skeleton' fragments exist after a long time, providing stability to the clusters. Our results support the previously proposed mechanism of crystallization. ${ }^{25}$ The crystallization of glycine could proceed through the formation of pre-nucleation clusters. These are thermodynamically stable solutes and there is no boundary between the clusters and the surrounding solution. They participate in the process of phase separation. The clusters can subsequently aggregate, leading to liquid-liquid separation. Nucleation of the crystalline phase then occurs within the dense hydrated amorphous phase. 


\section{Conclusions}

The properties of simulated systems depend on intermolecular electrostatic interactions. To reduce the influence of this factor on the results, three charge sets (B3LYP, mB3LYP and CNDO) coupled with the AMBER force field were selected and simulations of aqueous solutions were performed. All models predicted the density and diffusivity in agreement with data available in the literature. The properties of glycine clusters were studied. Despite small quantitative differences, they were the same in all these models.

Clustering rapidly increased with the increase in concentration of the solutions. In a concentrated aqueous solution, only a third of the glycine molecules were in a monomeric state. Percolated clusters are not observed even in a saturated solution, but an electric field facilitates cluster formation. Percolated chain-like and stripe-like glycine clusters are observed in the saturated aqueous solutions when a strong electric field was applied. This result supports the hypothesis for the orientational ordering of glycine clusters in the presence of a charged surfactant monolayer or an intense nanosecond near-infrared laser pulses with linear polarization of the laser beam (NPLIN phenomenon). Thus, an electric field can be used to control the nucleation and crystallization in saturated and supersaturated aqueous solutions of glycine. The orientational ordering inherent to the $\gamma$-polymorph appeared in a concentrated solution when a strong electric field was present. As a result, $\gamma$-glycine can be synthesised instead of the $\alpha$-polymorph.

The calculated average radii of gyration for the finite clusters are in agreement with the results of the SAXS experiments. The estimated fractal dimension of the clusters is about 2 . There is no phase boundary in the solutions. The mean residence lifetime of the water molecules from the hydration shells of the $-\mathrm{NH}_{3}$ and - $\mathrm{COO}$ groups is of the order of $10 \mathrm{ps;}$ in addition, short- and long-living glycine pairs were observed and some pairs moved together in the liquid for more than 1 ns. The presence of two mean lifetimes of glycine molecules demonstrates the lability and stability of the clusters. The clusters are highly dynamic objects that are in equilibrium with their monomers. The diffusivity of glycine in the solutions weakly decreases with increase in concentration and it is only two times smaller than the diffusivity of water. Changes in the shape and size of the clusters occur over hundreds of picoseconds. Thus, our simulations show that the glycine clusters are strongly hydrated solutes with a liquid-like character.

\section{Conflicts of interest}

There are no conflicts to declare.

\section{Acknowledgements}

This work was performed at the Institute of Chemistry of Macro Heterocyclic Compounds at Ivanovo State University of Chemistry and Technology. This work was supported by the
Russian Science Foundation under project number $14-23$ 00204 - C. The research is carried out using the equipment of the shared research facilities of HPC computing resources at Lomonosov Moscow State University [project number 1756]. DL_FIELD is a program package written by C. W. Yong and was obtained from STFC's Daresbury Laboratory via the website.

\section{Notes and references}

1 K. Srinivasan, K. Renuka Devi and S. Anbuchudar Azhagan, Cryst. Res. Technol., 2011, 46, 159-165.

2 R. A. Kumar, R. E. Vizhi, N. Vijayan and D. R. Babu, Phys. B, 2011, 406, 2594-2600.

3 G. L. Perlovich, L. K. Hansen and A. Bauer-Brandl, J. Therm. Anal. Calorim., 2001, 66, 699-715.

4 E. V. Boldyreva, V. A. Drebushchak, T. N. Drebushchak, I. E. Paukov, Y. A. Kovalevskaya and E. S. Shutova, J. Therm. Anal. Calorim., 2003, 73, 409-418.

5 E. V. Boldyreva, V. A. Drebushchak, T. N. Drebushchak, I. E. Paukov, Y. A. Kovalevskaya and E. S. Shutova, J. Therm. Anal. Calorim., 2003, 73, 419-428.

6 L. J. Little, R. P. Sear and J. L. Keddie, Cryst. Growth Des., 2015, 15, 5345-5354.

7 I. Weissbuch, V. Y. Torbeev, L. Leiserowitz and M. Lahav, Angew. Chem., Int. Ed., 2005, 44, 3226-3229.

8 Z. Liu, L. Zhong, P. Ying, Z. Feng and C. Li, Biophys. Chem., 2008, 132, 18-22.

9 B. A. Garetz, J. Matic and A. S. Myerson, Phys. Rev. Lett., 2002, 89, 175501.

10 X. Sun, B. A. Garetz and A. S. Myerson, Cryst. Growth Des., 2006, 6, 684-689.

11 J. E. Aber, S. Arnold, B. A. Garetz and A. S. Myerson, Phys. Rev. Lett., 2005, 94, 1-4.

12 K. Chadwick, R. J. Davey, R. Mughal and I. Marziano, Org. Process Res. Dev., 2009, 13, 1284-1290.

13 K. Allen, R. J. Davey, E. Ferrari, C. Towler, G. J. Tiddy, M. O. Jones and R. G. Pritchard, Cryst. Growth Des., 2002, 2, 523-527.

14 N. Takenaka, Y. Kitamura, Y. Koyano and M. Nagaoka, J. Chem. Phys., 2012, 137, 24501.

15 S. Gnanasambandam, Z. Hu, J. Jiang and R. Rajagopalan, J. Phys. Chem. B, 2009, 113, 752-758.

16 A. White and S. Jiang, J. Phys. Chem. B, 2011, 115, 660-667.

17 C. T. Andrews and A. H. Elcock, J. Chem. Theory Comput., 2013, 9, 4585-4602.

18 K. Leung and S. B. Rempe, J. Chem. Phys., 2005, 122, 184506.

19 M. J. Schnieders, J. Baltrusaitis, Y. Shi, G. Chattree, L. Zheng, W. Yang and P. Ren, J. Chem. Theory Comput., 2012, 8, 1721-1736.

20 Y. Yani, P. S. Chow and R. B. H. Tan, Cryst. Growth Des., 2012, 12, 4771-4778.

21 D. W. Cheong and Y. D. Boon, Cryst. Growth Des., 2010, 10, 5146-5158.

22 W. D. Cornell, P. Cieplak, C. I. Bayly, I. R. Gould, K. M. Merz, D. M. Ferguson, D. C. Spellmeyer, T. Fox, J. W. Caldwell and P. A. Kollman, J. Am. Chem. Soc., 1995, 117, 5179-5197. 
23 M. P. Kolesnikov, N. I. Voronova and I. A. Egorov, Origins Life, 1981, 11, 223-231.

24 J. Sun, D. Bousquet, H. Forbert and D. Marx, J. Chem. Phys., 2010, 133, 114508.

25 D. Gebauer, M. Kellermeier, J. D. Gale, L. Bergström and H. Cölfen, Chem. Soc. Rev., 2014, 43, 2348-2371.

26 W. Smith and T. R. Forester, J. Mol. Graphics, 1996, 14, 136-141.

27 S. L. Price, S. Hamad, A. Torrisi, P. G. Karamertzanis, M. Leslie and C. R. A. Catlow, Mol. Simul., 2006, 32, 985-997.

28 S. Melchionna, G. Ciccotti and B. L. Holian, Mol. Phys., 1993, 78, 533-544.

29 P. Raiteri and J. D. Gale, J. Am. Chem. Soc., 2010, 132, 17623-17634.

30 A. Bouchard, G. W. Hofland and G. J. Witkamp, J. Chem. Eng. Data, 2007, 52, 1626-1629.

31 T. Edvinsson, P. J. Råsmark and C. Elvingson, Mol. Simul., 1999, 23, 169-190.

32 Y. Wu, H. L. Tepper and G. A. Voth, J. Chem. Phys., 2006, 124, 24503.

33 J. L. Derissen, P. H. Smit and J. Voogd, J. Phys. Chem., 1977, 81, 1474-1476.

34 J. Voogd, J. L. Derissen and F. B. Van Duijneveldtt, J. Am. Chem. Soc., 1981, 103, 7701-7706.
35 T. S. Banipal, D. Kaur and P. K. Banipal, J. Chem. Eng. Data, 2004, 49, 1236-1246.

36 J. Huang, T. C. Stringfellow and L. Yu, J. Am. Chem. Soc., 2008, 130, 13973-13980.

37 W. S. Price, H. Ide and Y. Arata, J. Phys. Chem. A, 1999, 103, 448-450.

38 S. Hamad, C. E. Hughes, C. R. A. Catlow and K. D. M. Harris, J. Phys. Chem. B, 2008, 112, 7280-7288.

39 Y. Kameda, H. Ebata, T. Usuki, O. Uemura and M. Misawa, Bull. Chem. Soc. Jpn., 1994, 67, 3159-3164.

40 M. G. Campo, J. Chem. Phys., 2006, 125, 114511.

41 C. E. Hughes, S. Hamad, K. D. M. Harris, C. R. A. Catlow and P. C. Griffiths, Faraday Discuss., 2007, 136, 71-89.

42 M. Isichenko, Rev. Mod. Phys., 1992, 64, 961-1043.

43 D. Tiggemann, Int. J. Mod. Phys. C, 2006, 17, 1141-1150.

44 S. Chattopadhyay, D. Erdemir, J. M. B. Evans, J. Ilavsky, H. Amenitsch, C. U. Segre and A. S. Myerson, Cryst. Growth Des., 2005, 5, 523-527.

45 D. Erdemir, S. Chattopadhyay, L. Guo, J. Ilavsky, H. Amenitsch, C. U. Segre and A. S. Myerson, Phys. Rev. Lett., 2007, 99, 115702-115704.

46 Y. G. Bushuev and L. S. Bartell, J. Phys. Chem. B, 2007, 111, 1712-1720.

47 I. L. Dimitrov, F. V. Hodzhaoglu and D. P. Koleva, J. Biol. Phys., 2015, 41, 327-338. 